\title{
Tracheoesophageal fistula after esophageal atresia repair: recurrent, missed or acquired
}

\author{
Ozlem Boybeyi-Turer, Tutku Soyer \\ Department of Pediatric Surgery, Hacettepe University Faculty of Medicine, Ankara, Turkey \\ Contributions: (I) Conception and design: Both authors; (II) Administrative support: Both authors; (III) Provision of study materials or patients: \\ Both authors; (IV) Collection and assembly of data: Both authors; (V) Data analysis and interpretation: Both authors; (VI) Manuscript writing: Both \\ authors; (VII) Final approval of manuscript: Both authors. \\ Correspondence to: Ozlem Boybeyi-Turer, MD. Associate Professor of Pediatric Surgery, Department of Pediatric Surgery, Hacettepe University \\ Faculty of Medicine, Ankara, Turkey. Email: ozlemboy80@yahoo.com.
}

\begin{abstract}
Esophageal atresia (EA) is a common congenital anomaly of pediatric surgical practice. Tracheoesophageal fistula (TEF) is defined as congenital fistulous connection between esophagus and airway. All newborns with EA need immediate surgical repair of this anomaly. As the survival has reached to 95\%, the long-term consequences of TEF have become the focus of interest. The main complications of EA repair are classified as early complications including anastomotic leak, stricture, recurrent TEF (recTEF) and late complications including gastroesophageal reflux, esophageal dysmotility, tracheomalacia and respiratory disorders. TEFs seen after EA repair are the most challenging complication for both patient and the surgeon. These fistulas can occur after EA repair because of the recanalization of ligated original TEF called recTEF, a de novo fistula with different location called acquired TEF (acqTEF) or a second congenital fistula missed at the primary surgery called missed TEF (mTEF). Overall, incidence of fistula recurrence is between $5-10 \%$. The well-described risk factors for TEF recurrences are inadequate ligation of original TEF, anastomotic complications including leak, stricture and ischemia, tracheal injuries at the primary repair, and juxtapositioning of esophageal and tracheal suture lines. The clinical presentation of all types of fistulas is similar. Thus, the diagnosis of all these fistulas is challenging. Even, all patients having coughing, chocking or recurrent respiratory tract infections after EA repair should be investigated for the presence of TEF recurrence. In addition, the management of each type of fistula requires different approaches and can be technically demanding for most cases. Although surgical management is mostly inevitable in the management of recTEF, medical observation may be a choice for the management of acqTEFs since there is a possibility of spontaneous healing. Despite the advanced surgical technique, the risk for secondary recurrences, increased morbidity and even mortality are still exists. Therefore, this review will focus on all of the aspects of TEFs seen after EA repair.
\end{abstract}

Keywords: Esophageal atresia (EA); tracheoesophageal fistula (TEF); recurrent tracheoesophageal fistula (recTEF); missed tracheoesophageal fistula (mTEF); acquired tracheoesophageal fistula (acqTEF)

Received: 07 October 2020; Accepted: 30 October 2020; Published: 25 August 2022.

doi: $10.21037 /$ ccts-20-166

View this article at: http://dx.doi.org/10.21037/ccts-20-166 


\section{Introduction}

Esophageal atresia (EA) is a common and important congenital anomaly of pediatric surgical practice affecting one in 2,500 to 4,500 live births. Tracheoesophageal fistula (TEF) is defined as congenital fistulous connection between esophagus and airway. EA and TEF may be presented together or less commonly presented as separate malformations $(1,2)$. All newborns with EA need immediate surgical repair of this anomaly. Following the first successful repair of EA which was performed by Haight and Towsley in 1941 (3), the survival rate has improved up to $95 \%$ thank to advances in neonatal intensive care and anesthesia. As more patients reach adulthood, more studies focus on the long-term complications of EA repair.

The main complications of EA repair are classified as early and late complications. Anastomotic leak, stricture and recurrent TEF (recTEF) are the early complications, whereas gastroesophageal reflux, esophageal dysmotility, tracheomalacia and respiratory disorders are the late ones $(1,2,4,5)$. TEFs seen after EA repair are the most challenging complication for both patient and the surgeon. The fistulas between esophagus and airway can be congenital, recurrent, missed, or acquired. Not only the diagnosis of all these fistulas is difficult, but also the management is still challenging since secondary surgeries causes increased risk for secondary recurrences, increased morbidity and even mortality (6). In this review, it is aimed to discuss the diagnosis, treatment and outcome of all types of TEFs in children.

\section{Definition and classification of TEF}

The standard approach to the repair of EA and TEF is well described which consists of ligation of fistula and primary anastomosis of esophagus. Although EA repair has usually excellent outcome, several post-operative challenging situations may come through including fail of repair, need for lengthening procedures, need for revisional surgeries, and several defined complications (4,7). Among those, the fistula occurrence after primary repair of EA is one of the most challenging complications (4). A fistula can occur after EA repair because of the recanalization of ligated original TEF, a de novo fistula with different location or a second congenital fistula missed at the primary surgery (8). Overall, incidence of fistula recurrence is between $5-10 \%$ (9). Although the clinical presentations of these recurrent fistulas are similar, several differences might be existing regarding the management of these fistulas. Based on this point, Smithers et al. suggested a new classification of the fistulas occurred after EA repair (9). They classified postoperative fistulas as recTEF, missed TEF (mTEF) and acquired TEF (acqTEF) (9). RecTEF is defined as the fistula recurred at the original TEF location. MTEF is the second congenital fistula, actually a proximal fistula missed at the primary repair. AcqTEF is defined as a de novo fistula between esophagus and airway at a new location. AcqTEFs include all new connections between esophagus or colonic and gastric conduit and any level of airway such as trachea, bronchia, or pulmonary parenchyma (9). The acqTEFs secondary to EA-TEF repair will be discussed in this review. AcqTEFs due to cancer, trauma and other causes are not included in the scope of this review.

\section{$\operatorname{RecTEF}$}

Although the surgical outcome of EA and TEF repair is mostly satisfying, a small but important portion of patients needs secondary or revisional surgery. Most of these secondary surgeries are need to be performed for recurrent fistulas. Zhu et al. also reported that $76 \%$ of the patients with EA were re-operated for recTEF (10).

There are several well-described risk factors of recTEF. These risk factors can be listed as inadequate ligation of original TEF, anastomotic complications including leak, stricture and ischemia, tracheal injuries at the primary repair, and juxtapositioning of esophageal and tracheal suture lines $(1,6,11)$. It would be beneficial to be aware of these risk factors in order to decrease the recTEF incidence.

\section{Pathogenesis of recTEF}

In open repair of EA and TEF, the fistula is closed with sutures and divided which is an important step in preventing recurrence of fistula. However, in thoracoscopic repair, the fistula is usually ligated with titanium clips (12). A recent systematic review about thoracoscopic repair of EA revealed that the preferences for fistula ligation was suture in $30 \%$ of cases, and clips with/without sutures at the rest of the cases (12). In the same review, the endoclip displacement was seen in two of nine complicated cases, whereas fistula recurrence was seen in $5.3 \%$ of cases (12). On the other hand, Schlesinger et al. reported a case whose primary repair was performed thoracoscopically, and diagnosed as recTEF because of migrated clips (13). Although their recurrence rate is low, they emphasized the possibility of recTEF in cases with migrated clips in chest radiogram (13). 
Additionally, there are several studies reporting that there is no significant difference regarding recTEF incidence between open and thoracoscopic repair $(14,15)$.

The complications of esophageal anastomosis are important risk factors for fistula recurrence. Anastomotic leak after primary repair of EA possibly cause local or diffuse mediastinitis leading to breakdown of tracheal closure and causing recTEF $(14,16,17)$. In a retrospective cohort study, 26 cases with recTEF were reported and 18 of them (68\%) were reported to have experienced anastomotic leak after the primary repair (16). In a multi-institutional retrospective cohort study, recTEF occurred in $5 \%$ of the cases and univariate analysis revealed that anastomotic leak was significantly associated with increased risk of recTEF with OR of $4.26(1.47-12.3, \mathrm{P}=0.007)$ (14). The multivariate analysis in the same study also revealed significantly increased risk of recTEF in anastomotic leak with OR of 5.03 (95\% CI: 1.14-22.3, P=0.033) (14). Interestingly, the authors emphasized that placement of prosthetic material between suture lines increases anastomotic leak incidence, but did not cause difference in the incidence of recTEF (14).

The tissue damage at the anastomotic site is another important cause of recTEF. This tissue damage might be seen because of tissue ischemia, tensioned anastomosis, early or forced dilatation of esophageal strictures $(6,18)$. Briganti et al. also stated that esophageal dilatation in severe stricture may be a risk factor for recTEF (19). However, their results are not statistically significant. Although it is reasonable that aggressive dilatation might cause leaks, tissue damage and eventually recTEF, it seems that future prospective studies are needed to confirm this issue. Similarly, juxtapositioning of esophageal and tracheal suture lines is considered a risk factor for recurrence $(18,19)$. Therefore, the interposing various tissues such as pleura, pericardium, intercostals muscle are recommended to prevent recurrence of fistula $(6,18,19)$.

\section{Diagnosis of recTEF}

The diagnosis of recTEF is also challenging. The patients with EA very commonly experience several respiratory symptoms such as coughing, chocking or recurrent respiratory tract infections $(1,6,7,11)$. Since all of the patients either with gastroesophageal reflux, tracheomalacia or recTEF experience similar symptoms, it is difficult to distinguish which of the EA-related complications is the cause of these symptoms. Therefore, all patients having coughing, chocking or recurrent respiratory tract infections after EA repair should be investigated for the presence of
recTEF.

The diagnostic modalities include esophagogram and bronchoscopy $(6,7,11)$. The routine esophagogram may miss recTEF very commonly. Therefore, prone pullback method should be used to visualize a recTEF (11). The most appropriate way to visualize a recurrent fistula is to pass a tube down to esophagus and then to push the tube while giving contrast agent in prone position $(11,16)$. Nevertheless, both diagnosis and precise localization of the fistula could not be possible even with prone pullback esophagogram. Therefore, bronchoscopy should be performed to confirm the diagnosis and localize the fistula (16). Additionally, placing a catheter into the fistula through the working channel of the bronchoscope and then pulling it from the esophagus, and fixing it in place would provide an easier dissection of fistula during the surgery (16). In some occasions such as presence of very small pit or presence of irregular fistula tract due to increased fibrosis, catheterization of fistula would not be possible. In this case, methylene blue test, applying methylene blue from the opening and checking esophagus whether methylene blue passed or not, can be performed $(6,16,20)$. Most authors suggest using rigid bronchoscope in order to confirm diagnosis and place the guiding catheter. However, Hotta et al. suggested to perform flexible bronchoscopy and flexible esophagoscopy to localize the fistula in cases that exact diagnosis could not be achieved with rigid bronchoscopy (20).

\section{Management of recTEF}

Once the diagnosis of recTEF was made, historically open surgical repair was the only option for management. The open surgical management consists of localization, religation and division of the recurrent fistula and interposing a viable tissue between suture lines via thoracotomy (6). The periesophageal and peritracheal inflammation and fibrosis in the re-operated field makes the open repair challenging $(6,21)$. Waiting at least 5 weeks after the initial repair is recommended for resolution of the inflammation in the operation field (21).

Traditionally, open approach for recTEF is suggested performing via a right-sided thoracotomy since the recTEF is expected to be localized at the original TEF site $(7,21)$. However, in a few study, it has been reported that in cases with severe mediastinal inflammation and fibrosis, transtracheal route or median sternotomy route are alternative approaches (21-23). The esophageal replacement can also be performed in cases operated for several times and had multiple recurrences as a last choice of management (6). 
Table 1 The summary of open and endoscopic management approaches of recTEF (21,28-33)

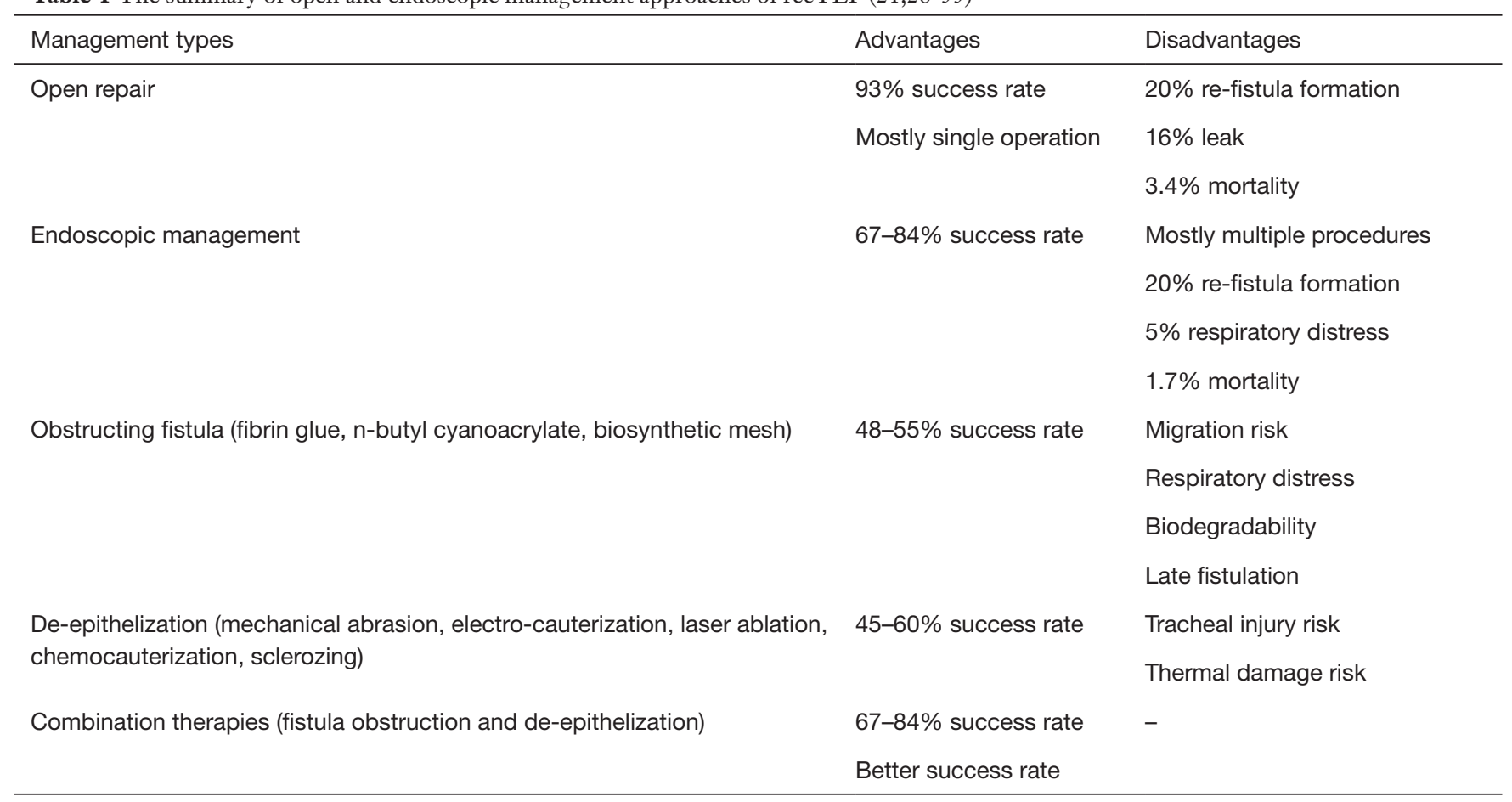

recTEF, recurrent tracheoesophageal fistula.

On the contrary, some authors encourage thoracoscopic approach for better visualization and accurate dissection in surgical management of recTEF (24). Since thoracoscopic approach has several advantages, it should be kept in mind especially for the patients whose primary repair was also performed thoracoscopically.

Many authors recommend interposing a viable tissue between suture lines in order to prevent a secondary recurrence of fistula $(6,21)$. Vascularized pleura, pericardium and intercostal muscle flaps are most commonly used tissues for interposing $(19,21)$. Moreover, costal cartilage graft could also be used especially in cases that were experienced tracheal injury in the initial surgery (25). The other rarely used are omental flap (26) and combination of free auricular and fascia lata graft (27). There is no literature data supporting one tissue in preventing fistula recurrence.

The major complications of open repair of recTEF are leak and secondary recurrence of TEF. The possibility of complications and rate of mortality increases especially in patients having associated esophageal stricture (7). In rare instances, various reconstructive procedures may be needed to restore the esophageal continuity. However, mostly open repair of recTEF has excellent outcomes, and usually does not require more than two times of re-operation (21).
The challenges in open repair of recTEF led many authors to perform endoscopic management as first line therapy of recTEF $(6,21)$. The endoscopic management techniques are classified into three main categories; obstruction of fistula tract, de-epithelization of the tract, combination of de-epithelization and obstruction of tract $(6,21)$. The commonly used agents for obstruction of tract are fibrin glue and n-butyl cyanoacrylate, and less commonly biosynthetic mesh $(21,28,29)$. The de-epithelization can be accomplished with mechanical abrasion, electrocauterization, laser ablation, chemocauterization and sclerosing (18,30-33). According to the literature, there is no superiority of any technique to another (6). All of these procedures require bronchoscopy with or without esophagoscopy. The patients should not be fed orally for a few days and control esophagogram should be undertaken before oral feeding (Table 1).

Although there is exceeding number of studies in the literature regarding the endoscopic management of recTEF, most of the studies are case series or retrospective studies with small number of subjects. The overall success rate ranging between $60 \%$ and $74 \%$, low complication rates and less morbidity, and easily re-applicability makes the endoscopic procedures preferred choice of management 


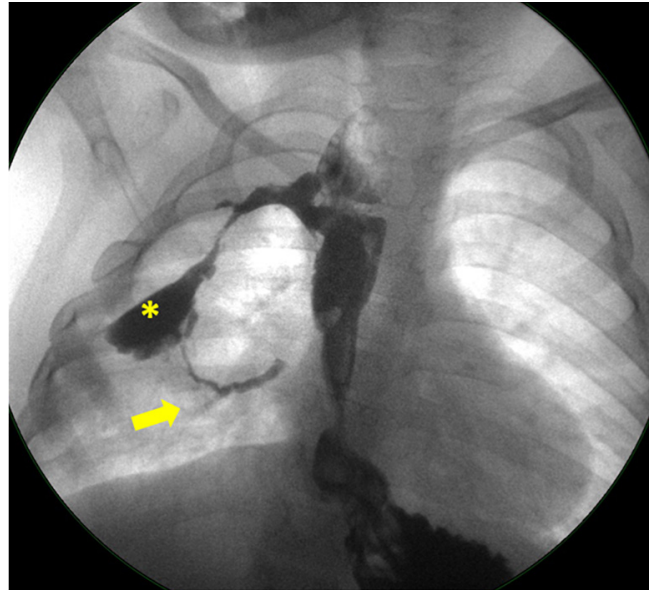

Figure 1 An example of acqTEF (authors' own patient): The esophagography revealing acquired fistula (arrow) between esophagus and right bronchus passing through the abscess pouch (asterisk). acqTEF, acquired tracheoesophageal fistula.

$(6,18,21)$. However, endoscopic procedures are not free of complication. The most terrifying complications are respiratory distress due to migration and aspiration of sealant or injury to trachea during cauterization $(21,29)$. In addition, multiple procedures are performed to achieve the aforementioned success rates (21). This brings multiple hospitalizations, increased cost, and increased pulmonary morbidity. A recent systematic review revealed that open repair has higher success rates, lower morbidity and mortality and less number of procedures than endoscopic approach (21). Although endoscopic treatments are good alternative for selected cases, the treatment failures should be considered.

\section{mTEF}

Persistent respiratory symptoms after EA repair are mostly caused by a recTEF. However, some of them are actually more proximal fistula missed at the primary repair of EA. According to the Gross classification of EA, the incidence of Gross type-A (isolated EA) is $8 \%$, type-B (proximal fistula with distal atresia) is $1 \%$ and the incidence of type-D (proximal and distal fistula) is $1 \%$. Notably, presence of a proximal fistula should not be underestimated especially in patients who were initially diagnosed as isolated EA (34). Even more, some authors reported the incidence of missed proximal fistula as $4 \%$ to $6 \%$ in their study $(34,35)$.

The pre-operative diagnosis of a proximal fistula is difficult, because the diagnosis of EA with distal fistulais usually made by a plain radiogram with nasogastric tube only or the amount of contrast is not suitable enough to fill the proximal fistula. Therefore, bronchoscopy is considered as the gold standard test in diagnosis of proximal fistula (34-36). During bronchoscopy, some identifying characteristics should be considered in order to make diagnosis properly (34). The proximal fistulas are usually located very close to vocal cords and have very small orifice, so a careful examination of upper trachea is mandatory (34). The best way to show a proximal fistula during bronchoscopy is insufflation of air from esophagus (34).

In recent studies, 3D CT and virtual bronchoscopy are reported to be useful in diagnosis of mTEF (37). Although it is a useful technique to confirm the diagnosis and show the concurrent problems such as tracheomalacia, the radiation risk is very high especially in pediatric population. Instead, performing bronchoscopy to all patients with EA is recommended to prevent delay in diagnosis.

Since mTEFs do not heal spontaneously, surgical management is required. Mostly, cervical approach may be needed because of the more proximal location-about T1 to T2 level—of these fistulas $(35,36)$.

\section{AcqTEF}

The definition 'acqTEF' is a little bit confusing since it has usually used to define the fistulas that are seen secondary to foreign body ingestion or iatrogenic trauma $(38,39)$. However, Smithers et al. classified the new TEF with a long and different location that is seen after EA repair as acqTEF (9). The location of these fistulas is different from the original TEF location. They have long new pathway extending from any part of airway including trachea, bronchia and lung parenchyma to esophagus or the replaced conduit $(9,40)$.

The diagnosis of acqTEF is challenging because of its unusual location (Figure 1). It is usually difficult to demonstrate the fistula and mostly impossible to cannulate during bronchoscopy because of tortuous and irregular border of fistula tract and abnormal location of the orifice (40).

Once the diagnosis is confirmed, the management of fistula should be performed surgically (9). However, there are some studies revealing that there is a possibility of spontaneous healing of acquired fistula $(38,40)$. The probable cause of spontaneous healing is thought to be due to ongoing inflammation ad fibrosis that helps to the closure of abnormally located fistula tract. Therefore, active 
observation for 6-12 weeks is recommended before surgical management in order to help healing and improve surgical dissection by allowing decrease in inflammation at the operation field $(38,40)$.

\section{Conclusions}

Based on this literature review, the patients having persistent respiratory symptoms should be examined for possible recTEF, mTEF and acqTEF. Although the clinical presentation of all post-operative TEF is similar, the diagnostic modalities and management approaches may vary. Therefore, a prompt diagnosis and accurate classification of post-operative TEF may improve longterm morbidity and mortality of these patients.

\section{Acknowledgments}

Funding: None.

\section{Footnote}

Provenance and Peer Review: This article was commissioned by the editorial office, Current Challenges in Thoracic Surgery, for the series "Tracheoesophageal Fistula". The article has undergone external peer review.

Conflicts of Interest: Both authors have completed the ICMJE uniform disclosure form (available at https://ccts. amegroups.com/article/view/10.21037/ccts-20-166/coif). The series "Tracheoesophageal Fistula" was commissioned by the editorial office without any funding or sponsorship. TS served as the unpaid Guest Editor of the series, and serves as an unpaid editorial board member of Current Challenges in Thoracic Surgery from March 2020 to February 2022. The authors have no other conflicts of interest to declare.

Ethical Statement: The authors are accountable for all aspects of the work in ensuring that questions related to the accuracy or integrity of any part of the work are appropriately investigated and resolved.

Open Access Statement: This is an Open Access article distributed in accordance with the Creative Commons Attribution-NonCommercial-NoDerivs 4.0 International License (CC BY-NC-ND 4.0), which permits the noncommercial replication and distribution of the article with the strict proviso that no changes or edits are made and the original work is properly cited (including links to both the formal publication through the relevant DOI and the license). See: https://creativecommons.org/licenses/by-nc-nd/4.0/.

\section{References}

1. Kovesi T, Rubin, S. Long-term complications of congenital esophageal atresia and/or tracheoesophageal fistula. Chest 2004;126:915-25.

2. Cartabuke RH, Lopez R, Thota PN. Long-term esophageal and respiratory outcomes in children with esophageal atresia and tracheoesophageal fistula. Gastroenterol Rep (Oxf) 2016;4:310-4.

3. Haight $\mathrm{C}$, Towsley HA. Congenital atresia of the esophagus with tracheoesophageal fistula: extrapleural ligation of fistula and end-to-end anastomosis of esophageal segments. Surg Gynecol Obstet 1943;76:672-88.

4. Teague WJ, Karpelowsky J. Surgical management of oesophageal atresia. Paediatr Respir Rev 2016;19:10-5.

5. Pinheiro PF, Simões e Silva AC, Pereira RM. Current knowledge on esophageal atresia. World J Gastroenterol 2012;18:3662-72.

6. Lal DR, Oldham KT. Recurrent tracheoesophageal fistula. Eur J Pediatr Surg 2013;23:214-8.

7. Koivusalo AI, Pakarinen MP, Lindahl HG, et al. Revisional surgery for recurrent tracheoesophageal fistula and anastomotic complications after repair of esophageal atresia in 258 infants. J Pediatr Surg 2015;50:250-4.

8. Nir V, Gur M, Toukan Y, et al. Factors associated with recurrence of tracheoesophageal fistula. Isr Med Assoc J 2018;20:687-90.

9. Smithers CJ, Hamilton TE, Manfredi MA, et al. Categorization and repair of recurrent and acquired tracheoesophageal fistulae occurring after esophageal atresia repair. J Pediatr Surg 2017;52:424-30.

10. Zhu H, Shen C, Xiao X, et al. Reoperation for anastomotic complications of esophageal atresia and tracheoesophageal fistula. J Pediatr Surg 2015;50:2012-5.

11. Coran AG. Redo esophageal surgery: the diagnosis and management of recurrent tracheoesophageal fistula. Pediatr Surg Int 2013;29:995-9.

12. Iacona RV, Saxena AK. Thoracoscopic repair of esophageal atresia with distal tracheoesophageal fistula (type C): systematic review. Surg Laparosc Endosc Percutan Tech 2020;30:388-93.

13. Schlesinger AE, Mazziotti MV, Cassady CI, et al. Recurrent tracheoesophageal fistula after thoracoscopic 
repair: vanishing clips as a potential sign. Pediatr Surg Int 2011;27:1357-9.

14. Lal DR, Gadepalli SK, Downard CD, et al. Challenging surgical dogma in the management of proximal esophageal atresia with distal tracheoesophageal fistula: Outcomes from the Midwest Pediatric Surgery Consortium. J Pediatr Surg 2018;53:1267-72.

15. Borruto FA, Impellizzeri $\mathrm{P}$, Montalto AS, et al. Thoracoscopy versus thoracotomy for esophageal atresia and tracheoesophageal fistula repair: review of the literature and metaanalysis. Eur J Pediatr Surg 2012;22:415-9.

16. Bruch SW, Hirschl RB, Coran AG. The diagnosis and management of recurrent tracheoesophageal fistulas. J Pediatr Surg 2010;45:337-40.

17. Friedmacher F, Kroneis B, Huber-Zeyringer A, et al. Postoperative complications and functional outcome after esophageal atresia repair: results from longitudinal singlecenter follow-up. J Gastrointest Surg 2017;21:927-35.

18. Meier JD, Sulman CG, Almond PS, et al. Endoscopic management of recurrent congenital tracheoesophageal fistula: a review of techniques and results. Int J Pediatr Otorhinolaryngol 2007;71:691-7.

19. Briganti V, Mangia G, Ialongo P, et al. Usefulness of large pleural flap for the treatment of children with recurrent tracheoesophageal fistula. Pediatr Surg Int 2009;25:587-9.

20. Hotta Y, Uezono S, Segawa O, et al. Precise localization of a recurrent tracheo-oesophageal fistula using retrograde guide wire placement. Paediatr Anaesth 2002;12:541-3.

21. Aworanti O, Awadalla, S. Management of recurrent tracheoesophageal fistulas: a systematic review. Eur J Pediatr Surg 2014;24:365-75.

22. Martin LW, Cox JA, Cotton R, et al. Transtracheal repair of recurrent tracheoesophageal fistula. J Pediatr Surg 1986;21:402-3.

23. Holland AJ, Ford WD, Guerin RL. Median sternotomy and use of a pedicled sternocleidomastoid muscle flap in the management of recurrent tracheoesophageal fistula. J Pediatr Surg 1998;33:657-9.

24. Cano Novillo I, Aneiros Castro B, Godoy Lenz J, et al. Thoracoscopic approach for complications after esophageal atresia repair: initial experience. Asian J Endosc Surg 2020;13:147-51.

25. Delarue A, Paut O, Simeoni J, et al. Costal cartilage grafting for repair of a recurrent tracheoesophageal fistula in a 1.6-kg baby with esophageal atresia. Pediatr Surg Int
2002;18:162-4.

26. Mangat S, Haithcock BE, McLean SE. Omental flap provides definitive management for pediatric patient with multiple tracheoesophageal fistula recurrences. Am Surg 2020;86:1553-5.

27. Sugiyama A, Urushihara N, Fukumoto K, et al. Combined free autologous auricular cartilage and fascia lata graft repair for a recurrent tracheoesophageal fistula. Pediatr Surg Int 2013;29:519-23.

28. Gutierrez C, Barrios JE, Lluna J, et al. Recurrent tracheoesophageal fistula treated with fibrin glue. J Pediatr Surg 1994;29:1567-9.

29. Keckler SJ, St Peter SD, Calkins CM, et al. Occlusion of a recurrent tracheoesophageal fistula with surgisis. J Laparoendosc Adv Surg Tech A 2008;18:465-8.

30. Gregory S, Chun RH, Parakininkas D, et al. Endoscopic esophageal and tracheal cauterization for closure of recurrent tracheoesophageal fistula: a case report and review of the literature. Int J Pediatr Otorhinolaryngol 2017;98:158-61.

31. Lelonge Y, Varlet F, Varela P, et al. Chemocauterization with trichloroacetic acid in congenital and recurrent tracheoesophageal fistula: a minimally invasive treatment. Surg Endosc 2016;30:1662-6.

32. Rakoczy G, Brown B, Barman D, et al. KTP laser: an important tool in refractory recurrent tracheo-esophageal fistula in children. Int J Pediatr Otorhinolaryngol 2010;74:326-7.

33. Sung MW, Chang H, Hah JH, et al. Endoscopic management of recurrent tracheoesophageal fistula with trichloroacetic acid chemocauterization: a preliminary report. J Pediatr Surg 2008;43:2124-7.

34. Summerour V, Stevens PS, Lander AD, et al. Characterization of the upper pouch tracheooesophageal fistula in oesophageal atresia. J Pediatr Surg 2017;52:231-4.

35. Parolini F, Morandi A, Macchini F, et al. Esophageal atresia with proximal tracheoesophageal fistula: a missed diagnosis. J Pediatr Surg 2013;48:E13-7.

36. Guo W, Li Y, Jiao A, et al. Tracheoesophageal fistula after primary repair of type $\mathrm{C}$ esophageal atresia in the neonatal period: recurrent or missed second congenital fistula. J Pediatr Surg 2010;45:2351-5.

37. Islam S, Cavanaugh E, Honeke R, et al. Diagnosis of a proximal tracheoesophageal fistula using threedimensional CT scan: a case report. J Pediatr Surg 2004;39:100-2.

38. Nazir Z, Khan MAM, Qamar J. Recurrent and acquired 
tracheoesophageal fistulae (TEF)-minimally invasive management. J Pediatr Surg 2017;52:1688-90.

39. Thakkar HS, Hewitt R, Cross K, et al. The multidisciplinary management of complex congenital and acquired tracheo-oesophageal fistulae. Pediatr Surg Int

doi: $10.21037 /$ ccts-20-166

Cite this article as: Boybeyi-Turer O, Soyer T. Tracheoesophageal fistula after esophageal atresia repair: recurrent, missed or acquired. Curr Chall Thorac Surg 2022;4:26.
2019;35:97-105.

40. Boybeyi Türer Ö, Tanyel FC, Soyer T. Acquired tracheoesophageal fistula after esophageal atresia repair. Balkan Med J 2019;37:47-9. 